\title{
Original
}

\section{El desarrollo de habilidades profesionales en los estudiantes de la carrera de Agronomía}

$$
\text { (Original) }
$$

\section{Development of professional skills in students of Agricultural science (Original)}

MSc. María Elena Sol-Victortes, Centro Universitario Municipal de Niquero, Cuba, msolv@udg.co.cu

MSc. Caridad Ixora Blanco-Figueredo, Centro Universitario Municipal de Niquero, Cuba, Iblancof@udg.co.cu

Recibido: 9/02/2018 Aceptado: 7/04/2018

\section{RESUMEN}

El artículo aborda la necesaria reflexión sobre el desarrollo de habilidades profesionales en los estudiantes de Agronomía para favorecer su educación, pues la misma se relaciona de forma significativa con muchos de los factores que sustentan la vinculación de la escuela con la vida y el estudio con el trabajo, a partir de los contenidos relacionados con diferentes asignaturas. Se trabajó con una población de 26 estudiantes tomándose como muestra 15 estudiantes, de ellos 8 hembras y 7 varones en edades aproximadas de 15 a 18 años. Por ello se proponen actividades para desarrollar habilidades profesionales teniendo en cuenta que la Agronomía es la especialidad de mayor prioridad en la enseñanza técnico profesional, pues prepara la fuerza técnica que producirá alimentos para el país desde el punto de vista técnico económico, y se debe salvaguardar como parte estratégica de la continuidad y sostenibilidad de la Revolución.

PALABRAS CLAVES: habilidades profesionales; Agronomía; sostenibilidad; producción de alimentos

\section{ABSTRACT}

The article addresses the necessary reflection on the development of professional skills in students of Agronomy to promote their education, as it is significantly related to many of the factors that support the link between school and life and study with the work, from the contents related to different subjects. It was used a population of 26 students, taking as sample 15 students, of them 8 females and 7 males in approximate ages of 15 to 18 years. Therefore, there are proposed activities to develop professional skills, taking into account that Agronomy is the specialty of highest priority in professional technical education, because it prepares the 
technical force that will produce food for the country, from the technical economic point of view, and it must be safeguarded as strategic part of the continuity and sustainability of the Revolution.

KEYWORDS: professional skills; Agronomy; sustainability; food production

\section{INTRODUCCIÓN}

La Revolución Cubana ha producido la más profunda transformación educacional que se conozca en los países de nuestra América. El Estado cubano, con la participación y respaldo de las organizaciones políticas y de masas, es el encargado de la reestructuración y funcionamiento de un sistema nacional de educación orientado al desarrollo y formación de las nuevas generaciones, constituyendo la Enseñanza Técnica y Profesional (E.T.P) un subsistema caracterizado por su constante perfeccionamiento, y significativo es el papel protagónico que asume esta enseñanza en la formación de la fuerza laboral nacional cubana.

La dinámica actual en los procesos productivos se caracteriza por la rápida incorporación de la alta tecnología, por las altas funciones en los oficios y por la movilidad en el campo de aplicación de los conocimientos, habilidades y valores, por ende, se requiere de un profesional competente capaz de enfrentar estos nuevos retos. La enseñanza técnica y profesional tiene el encargo social de formar estos trabajadores; actos para un mundo laboral en continuo cambio, donde se requiere actualizar las habilidades profesionales, cumpliendo la consigna de producir más con menos.

La Agronomía se encuentra dentro de las prioridades dentro de la enseñanza técnico profesional, es la que prepara la fuerza capaz de desempeñar en el país el reto de la producción agropecuaria integral, constituye una forma de organización y planificación de tareas docente-productivas encaminadas al logro de los conocimientos, hábitos, capacidades y habilidades profesionales para su futuro despeño profesional.

Hasta la actualidad se han desarrollado una serie de investigaciones, dentro y fuera de Cuba, que enfocan sus estudios hacia el desarrollo de las habilidades profesionales, en sentido general describen al proceso pedagógico profesional perteneciente a la educación técnica y profesional entre los que se destacan Bermúdez (2003), Alvares (1999), Patiño (1996), Abreu (1997), Cortijo (1996), López (2005), Marqués (1999), Rico (1990), Vargas (2001), Bell (2001), Addine (2004), entre otros. Estos investigadores conceden gran importancia al desarrollo de las habilidades profesionales. 
El vínculo entre lo profesional y la carrera se brinda por medio de la profesionalización de la formación básica y técnica en el Proceso Pedagógico Profesional, se interpreta como tendencia contemporánea o como enfoque del método para elevar la calidad de este proceso Abreu, (1997).

Sin embargo, resultan insuficientes los fundamentos y metodologías que orienten el trabajo con las habilidades profesionales desde el proceso pedagógico, en estrecha unidad con el tratamiento metodológico de las mismas.

Por ello es necesario favorecer el desarrollo de habilidades profesionales en los estudiantes de la carrera de Agronomía. Es incuestionable que para lograr la formación de un técnico se necesita contar con un sistema formativo que desarrolle determinadas cualidades, las que no distan de la concepción actual del modelo educativo cubano, no obstante, necesitado de modificaciones en su diseño y en su dinámica se centra en el Proceso Pedagógico Profesional en la asignatura Trabajo en la Producción Agropecuaria durante el segundo año. La investigación está basada en elaborar un sistema de actividades para favorecer el desarrollo de la habilidades profesionales, sustentado en la vinculación de la escuela con la vida y el estudio con el trabajo a partir de los contenidos relacionados con la asignatura Trabajo en la Producción Agropecuaria para favorecer el desarrollo de las habilidades profesionales en los estudiantes de segundo año de la especialidad Agronomía en la E.T.P. Se sustenta en el desarrollo de las habilidades en la asignatura Trabajo en la Producción Agropecuaria.

\section{POBLACIÓN Y MUESTRA}

Se trabajó con una población de 26 estudiantes tomándose como muestra 15 estudiantes de ellos 8 hembras y 7 varones en edades aproximadas de 15 a 18 años de la carrera de Agronomía de segundo año, perteneciente al I.PI "2 de Diciembre".

Los principales resultados radican en el proceso pedagógico profesional donde resulta imprescindible la formación de modos de actuación y un pensamiento divergente, crítico y reflexivo donde se reconoce el carácter activo y participativo del estudiante. En este proceso pedagógico se forman las habilidades profesionales que los estudiantes deben asumir en todas las actividades que realicen, donde el profesor debe analizar la estructura de las actividades (propuestas) a desarrollar y debe tener claridad de qué acciones y operaciones se forman en la misma, que sea del dominio de los fundamentos de la tecnología y que aprendan a pensar y trabajar con autonomía, adaptándose con más facilidad y creatividad a los más variados ambiente y situaciones del progreso y los cambios que se producen que pueden ser tipificados en tácticos, conceptuales y relacionales. 
Para analizar mejor los resultados se plantean tres momentos principales en el proceso:

1- Conocimiento e identificación de las habilidades: los estudiantes hacen referencia a una y otra habilidad y asumen la que puede incidir o la que le sea necesaria en su futura labor profesional. Son capaces de explicar la importancia de su estudio para desarrollar la habilidad.

2- Formación y desarrollo de las habilidades: se realizan actividades con diferentes niveles de complejidad donde los participantes se sienten protagonistas del proceso de formación de habilidades profesionales. Es determinante en este momento la labor del profesor al trabajar en función del aprendizaje por descubrimiento al tener en cuenta la zona de desarrollo próximo con el fin de incrementar la complejidad y la interacción de los estudiantes en las diferentes esferas productivas.

3 - Consolidación de la habilidad: es relevante destacar la calidad e influencia que ha ejercido el conocimiento y la utilización de las habilidades profesionales para su posterior aplicación de forma práctica en aras de producción y entidades productivas así como la necesidad de retroalimentarse a través de conocimientos teóricos que le son necesarios en la reinserción laboral.

Las reflexiones educativas que se efectuaron tuvieron un objetivo formativo importante, el conocimiento formación y desarrollo de habilidades profesionales a su vez, y valores necesario como son: la responsabilidad, honradez, laboriosidad y honestidad, decisivos para su desempeño futuro.

Valoración de la pertinencia de la investigación a través del método de evaluación por criterio de expertos.

Antes de llevar la investigación a la práctica pedagógica, se valoró su grado de aceptación por una parte de la comunidad científica especializada, a través del método de evaluación por criterio de expertos. Para ello, se sometieron a criterio los siguientes aspectos:

Las acciones, actividades y ejercicios que estructuran y armonizan la investigación brindan posibilidades de generalización en la práctica pedagógica del desarrollo de las habilidades profesionales. La valoración sobre la efectividad que tendrán las actividades en la práctica laboral.

Asimismo, se tuvieron en cuenta otros elementos que posibilitaron la aplicación del método empírico sustentado en las consultas a expertos. Los resultados del ordenamiento realizado por cada experto arrojaron que los elementos sometidos a criterios se ubican entre las categorías 5 (Bueno) y 7 (Excelente), lo que permite inferir que la investigación realizada es efectiva en la práctica pedagógica. 
Características del sistema de actividades como resultado científico.

Para darle solución a la problemática presente en la investigación se tiene en cuenta la línea trazada por el Ministerio de Educación referente a la Educación Técnica y Profesional en cuanto a: las habilidades profesionales, sus cualidades e influencia en la formación del técnico en Agronomía.

Las actividades realizadas son vías imprescindibles para la formación de las habilidades y capacidades de los estudiantes.

El presente sistema está formado por 12 actividades. Para su elaboración se tuvieron en cuenta los intereses y motivaciones de los estudiantes objeto de investigación y sus características psicopedagógicas.

Los autores asumen en la presente investigación el sistema de actividades como vía para favorecer el desarrollo de las habilidades profesionales por lo que considera que un sistema de condiciones interrelacionadas, con objetivos, etapas y metodologías precisas conforman el desarrollo de las habilidades profesionales.

Por lo que:

- Se elabora para solucionar problemas de la práctica y vencer dificultades con optimización de tiempo y recursos.

- Permite proyectar un cambio cualitativo y cuantitativo en el proceso a partir de eliminar las contradicciones entre el estado actual y el deseado.

- Implica un proceso de planificación en el que se produce el establecimiento de secuencias de acciones orientadas hacia el fin a alcanzar, lo cual no significa un único curso de las mismas.

- Interrelaciona dialécticamente en un plan global los objetivos o fines que se persiguen y la metodología para alcanzarlos.

- Fue concebido con enfoque sistémico en el que predominan las relaciones de coordinación, aunque no dejan de estar presentes las relaciones de subordinación, dependencia y jerarquía.

- Posee una estructuración a partir de fases o etapas relacionadas con las acciones de orientación, ejecución y control.

- Es un aporte eminentemente práctico debido a sus persistentes grados de utilidad.

- Tiene carácter dialéctico concedido por la búsqueda de cambio cualitativo que se producirá en el objeto (estado real a estado deseado), por las constantes adecuaciones 
que puede sufrir su accionar y por la articulación entre los objetivos (metas perseguidas) y la metodología (vías instrumentadas para alcanzarlas), entre otras.

- El hecho de responder a una contradicción entre el estado actual y el deseado de un objeto concreto ubicado en el espacio y en el tiempo que se resuelve mediante la utilización programada de determinados recursos y medios.

Las autoras para conformar el desarrollo de las habilidades profesionales tuvieron en cuenta los siguientes elementos:

- Existencia de insatisfacciones respecto a los fenómenos, objetos o procesos educativos en un contexto o ámbito determinado.

- Diagnóstico de la situación.

- Planteamiento de objetivos y metas a alcanzar en determinados plazos de tiempo.

- Definición de actividades y acciones que respondan a los objetivos trazados y entidades responsables.

- Planificación de recursos y métodos para viabilizar la ejecución.

- Evaluación de resultados.

Las autoras utilizarán la siguiente metodología para la aplicación del desarrollo de las habilidades: con anterioridad distribuirá una guía con las actividades a realizar. Las actividades serán distribuidas por equipos teniendo en cuenta el diagnóstico aplicado, para darles participación a aquellos estudiantes con dificultades en el desarrollo de las habilidades.

El componente ideo político en las actividades no debe faltar, por lo que se deben emplear preguntas dirigidas a contribuir al trabajo formativo.

La evaluación se realizará a partir de las mismas actividades.

La implementación de la metodología en la práctica pedagógica se realiza a través del método del experimento, en su variante de pre-experimento. Los resultados obtenidos demuestran la validez de la propuesta, se logró que el $100 \%$ de los estudiantes utilizaran correctamente las habilidades profesionales.

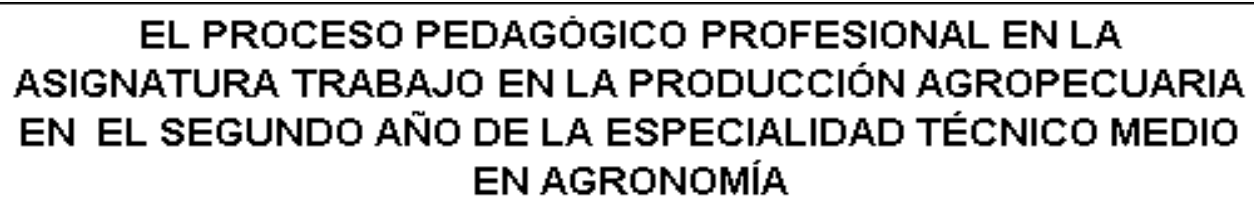

EL PROCESO PEDAGÓGICO PROFESIONAL EN LA ASIGNATURA TRABAJO EN LA PRODUCCIÓN AGROPECUARIA EN EL SEGUNDO AÑO DE LA ESPECIALIDAD TÉCNICO MEDIO EN AGRONOMÍA

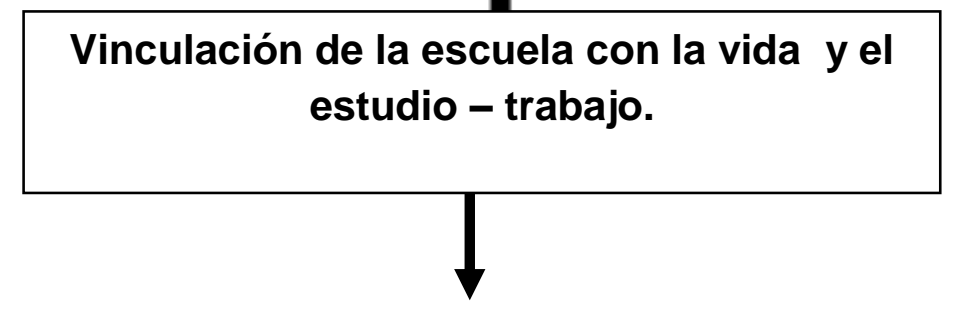




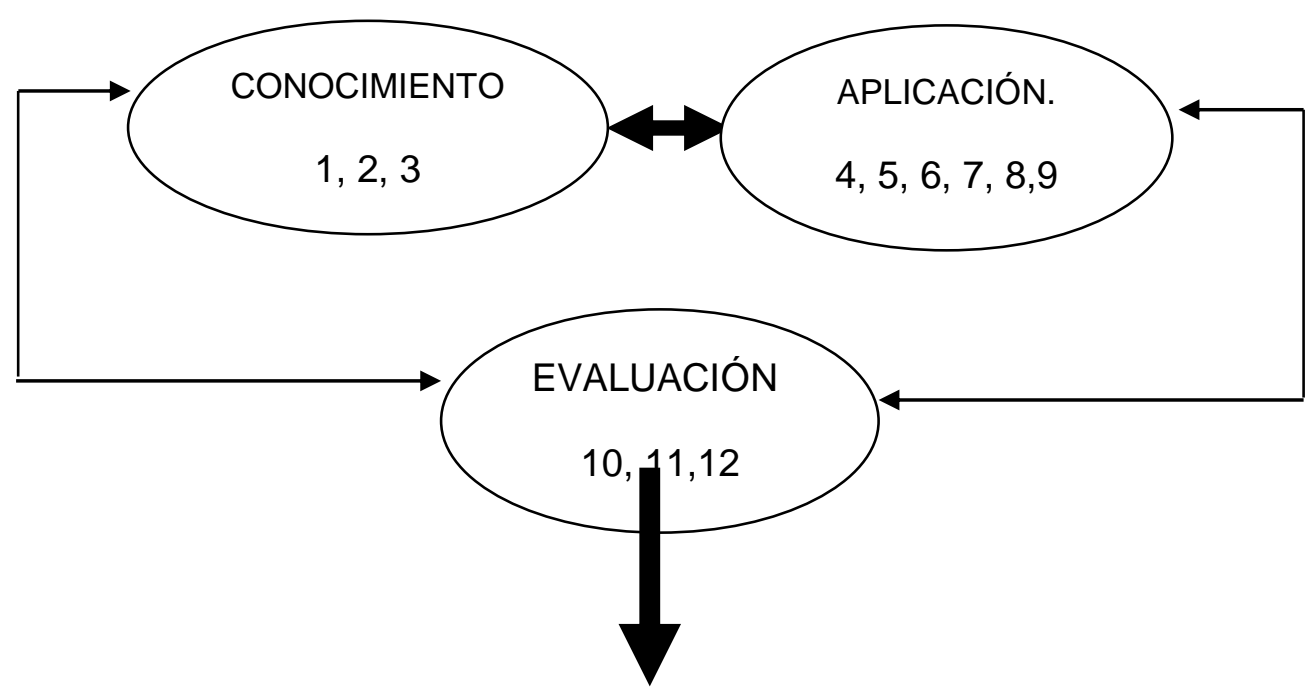

\section{EL DESARROLLO DE LA HÁBILIDAD IDENTIFICAR INSECTOS}

\section{ANÁLISIS DE LOS RESULTADOS}

Con vistas a obtener resultados positivos se realizaron preparaciones metodológicas en el departamento que sirvieron no solo para el profesor, sino también para el resto del colectivo, con el objetivo de que los mismos alcanzaran un nivel teórico y metodológico con relación al sistema de actividades como vía para el desarrollo de las habilidades profesionales y a la vez socializar la misma.

El diseño seleccionado para el pre-experimento consistió en la aplicación de una prueba pedagógica de entrada y de salida, concebida la misma para el momento inicial y final, una guía de observación a clase y el análisis de los resultados de la actividad desarrollada.

Se precisan las categorías en que quedarán evaluados los indicadores y se establece la escala de valoración, la que permitirá emitir juicios de valor del estado de las habilidades estudiadas. El nivel de desarrollo de las habilidades profesionales fue evaluado a través de los indicadores: rapidez, independencia, transferencia, precisión y conocimiento, en función del aprendizaje como una escala valorativa de BIEN (B); REGULAR (R); MAL (M).

A continuación se declara la manera de ubicar cada indicador en alguna de las categorías. Indicadores:

Escala.

Rapidez: nivel de desarrollo de las actividades durante la clase.

- Muy rápido: si realiza la actividad antes del tiempo establecido.

- Rápido: si realiza la actividad en el tiempo que establece el profesor. 
- Lento: si no logra realizar la actividad en el tiempo establecido.

Independencia: cuando se logran resolver las actividades de una forma Independiente.

- Si resuelve la actividad por sí solo.

- Si resuelve la actividad con ayuda de los estudiantes o del profesor o de Ambos.

$\mathrm{R}$

- Si no resuelve la actividad por sí solo, ni con ayuda.

$\mathrm{M}$

Transferencia: cuando los conocimientos, la sistematización, integración y generalización de los mismos es posible lograrla en todos los estudiantes que posean un nivel de razonamiento normal, siempre que se creen las condiciones docentes necesarias y se ejercite lo suficientemente hasta convertirlo en una práctica habitual, llegando los estudiantes a concientizar que a partir de los contenidos se puede llegar a obtener nuevos conocimientos.

- Si aplica de una forma creativa los conocimientos a nuevas situaciones.

$\mathrm{B}$

- Si reproduce a nuevas situaciones los conocimientos ya adquiridos.

$\mathrm{R}$

- Si no aplica a nuevas situaciones los conocimientos ya adquiridos.

$\mathrm{M}$

Precisión: evidencia el dominio de los conocimientos y de las acciones en el desarrollo de las actividades.

- Muy preciso: si logra realizar todas las acciones.

- Preciso: si logra realizar 3 - 4 acciones.

$\mathrm{R}$

- Impreciso: si logra realizar 1 - 2 o ninguna acción.

M

También se le otorgó una calificación para el análisis del desarrollo de las habilidades profesionales.

- Desarrollada: cuando cumple con 4 a 5 indicadores.

- En desarrollo: cuando cumple con 3 indicadores.

- No desarrollada: cuando cumple con menos de 3 indicadores

Este posee una concepción con enfoque sistémico en el que predominan las relaciones de coordinación, aunque no dejan de estar presentes las relaciones de subordinación, dependencia y jerarquía.

Prueba de Entrada.

Se realizó una prueba de entrada, con el fin de comprobar el desarrollo de las habilidades profesionales en la especialidad Agronomía. Para su evaluación, se tuvieron en cuenta cinco indicadores: rapidez, precisión, independencia, transferencia y conocimiento. Los resultados obtenidos fueron críticos, pues en el indicador rapidez el $40 \%$, obtuvo calificación de mal y el 
$26,66 \%$ de bien. En la independencia el $46.6 \%$ obtuvo mal y el $33.33 \%$, de bien. En la precisión el $46.66 \%$, mal y el $33.3 \%$, de bien. En la transferencia el $53.33 \%$ de mal y el 13.33 $\%$, de bien. En el conocimiento el $53.33 \%$, obtuvo mal y el $26.66 \%$, de bien.

Tabla 1 Resultados de la prueba de entrada

\begin{tabular}{|l|c|l|c|c|c|c|c|c|}
\hline Indicadores. & Bien & $\%$ & Regular & $\%$. & Mal & $\%$. & $\begin{array}{l}\text { Total } \\
\text { Inicial }\end{array}$ & $\%$. \\
\hline Rapidez. & 4 & 26,66 & 5 & 33,33 & 6 & 40 & 15 & 100 \\
\hline Independencia & 5 & 33,33 & 3 & 20 & 7 & 46,66 & 15 & 100 \\
\hline Precisión. & 5 & 33,33 & 3 & 20 & 7 & 46,66 & 15 & 100 \\
\hline Transferencia. & 2 & 13,33 & 5 & 33,33 & 8 & 53,33 & 15 & 100 \\
\hline Conocimiento. & 4 & 26,66 & 3 & 20 & 8 & 53,33 & 15 & 100 \\
\hline
\end{tabular}

Prueba de salida.

Se realizó una prueba con el fin de comprobar el desarrollo de las habilidades profesionales. Para la evaluación de la prueba de salida se tuvieron en cuenta cinco indicadores: rapidez, independencia, precisión, transferencia y conocimiento. Los resultados obtenidos demuestran que ha ido evolucionando, pues en el indicador rapidez 4 estudiantes alcanzaron calificación de regular, 10 de bien y 1 de mal; en la independencia 3 de regular, 11de bien y 1 de mal, en la precisión 10 de bien, 3 de regular y 2 mal; en la transferencia 12de bien, 2 de regular y 1 de mal y en el conocimiento 14 de bien, 1 de regular y 0 de mal.

Tabla 2. Prueba de salida

\begin{tabular}{|l|c|c|c|c|c|c|c|c|}
\hline Indicadores. & Bien & $\%$. & Regular & $\%$. & Mal & $\%$. & $\begin{array}{l}\text { Total } \\
\text { Inicial }\end{array}$ & $\%$. \\
\hline Rapidez. & 10 & 66.66 & 4 & 26,66 & 1 & 6,6 & 15 & 100 \\
\hline Independencia & 11 & 73,33 & 3 & 20 & 1 & 6,6 & 15 & 100 \\
\hline Precisión. & 10 & 66,66 & 3 & 20 & 2 & 13,33 & 15 & 100 \\
\hline Transferencia. & 12 & 80 & 2 & 13,33 & 1 & 6,66 & 15 & 100 \\
\hline Conocimiento. & 14 & 93,33 & 1 & 6,66 & 0 & - & 15 & 100 \\
\hline
\end{tabular}

Comparación entre la prueba de entrada y salida atendiendo a la categoría de Bien. 


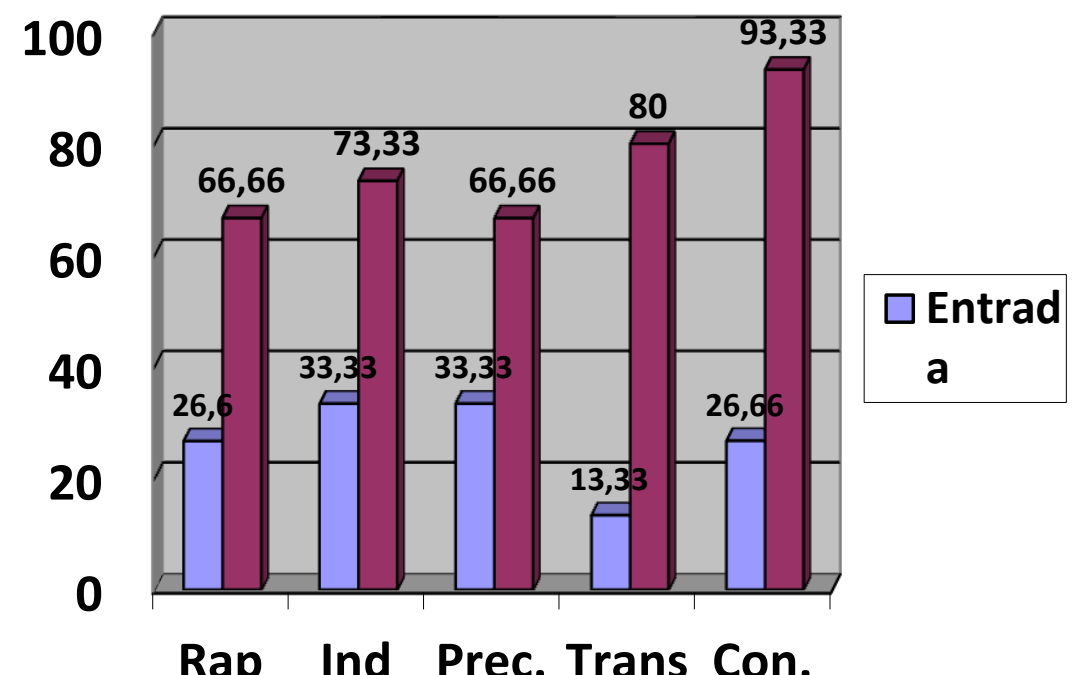

Fig. Comprobación entre la prueba de entrada y de salida

Estos importantes aspectos demuestran que los estudiantes han sabido identificar, formar, desarrollar y consolidar los indicadores de la habilidad "identificar insectos" y de hecho, aplicarlos a situaciones de su vida profesional y laboral en correspondencia con los modos de actuación de cada uno.

Al comparar los resultados obtenidos en el diagnóstico inicial y final se aprecia la efectividad de la aplicación práctica de las habilidades, en la constatación inicial en los estudiantes se manifestaba insuficiencias tales como: desconocimiento de las habilidades profesionales fundamentalmente las de identificar, analizar, caracterizar, clasificar comparar, insuficiente utilización de las habilidades en la práctica laboral.

Los resultados obtenidos evidencian avances en el desarrollo de las habilidades profesionales así como la preparación para su futuro desempeño. Entre los más significativos se destacan los siguientes:

Las actividades realizadas son vías imprescindibles para la formación de las habilidades y capacidades de los estudiantes, así como intereses y motivaciones como futuros profesionales.

\section{CONCLUSIONES}

1. Se evidenciaron insuficiencias significativas a partir de la prueba de entrada, lo que limita una correcta formación profesional del Técnico en la especialidad Agronomía, relacionadas con el desarrollo de las habilidades profesionales.

2. La aplicación de las actividades propuestas trasciende lo tradicional del Proceso Pedagógico Profesional de la asignatura Trabajo en la Producción Agropecuaria relacionado con las habilidades profesionales al revelar un aprendizaje desarrollador. 
3. El conocimiento y desarrollo de las habilidades profesionales permitió un cambio cualitativo y cuantitativo en el proceso pedagógico desde una dimensión educativa por la necesidad de preparar al hombre de vivir en una sociedad de ser productores y no consumidores.

\section{REFERENCIAS BIBLIOGRÁFICAS}

Abreu Regueiro, R. (1997). La pedagogía profesional un imperativo de la escuela y la empresa contemporánea. Tesis de Maestría en Pedagogía Profesional, ISPETP.La .La Habana.

Addine Fernández, F (2004).Didáctica, teoría y práctica .La Habana .Pueblo y Educación.

Álvarez Valiente, I. (1999). El proceso y sus movimientos: Modelo de la dinámica del proceso docente educativo en la educación superior. Tesis en opción al grado científico de Doctor en Ciencias Pedagógicas. Santiago de Cuba.

Álvarez de Zayas, C. (1999). La pedagogía como ciencia. La Habana: Félix Varela

Bell Rodríguez, R. y otros. (2001). Pedagogía y diversidad. La Habana: Abril.

Bermúdez Morris, R., Pérez Martin L. (2003). Aprendizaje y crecimiento personal. La Habana.

Cortijo Jacomino, R. (1996). Didáctica de las ramas técnicas, una alternativa para su desarrollo.

La Habana (en soporte electrónico).

López Espinosa, E. (2005). Consideraciones generales acerca de las habilidades. La Habana (en soporte electrónico).

Márquez Rodríguez, A. (1999). Habilidades reflexiones y reposiciones para su evaluación. Santiago de Cuba (en soporte electrónico).

Patiño Rodríguez, M., Hernández Hernández, A.M. León Consuegra, O. (1996). El modelo de la escuela politécnica cubana. Una realidad. La Habana: Pueblo y Educación.

Rico Montero, P. (1990). ¿Cómo desarrollar en los alumnos las habilidades para el control y la valoración de su trabajo docente? La Habana: Pueblo y educación.

Vargas Galarraga, A.H. (2001). Propuesta del rediseño del sistema de habilidades profesionales del perfil técnico de la disciplina Maquina y Accionamiento Eléctricos de la carrera licenciatura en educación en la especialidad Eléctrica. Tesis en opción al grado Académico de master en pedagogía profesional, instituto superior pedagógico. E.T.P. Hector A Pineda .Habana.

Roblejo Yero, Juan G. (2009). Sistema de actividades para favorecer el desarrollo de la habilidad roturar suelos, en los estudiantes de $3^{\circ}$ año de la especialidad Agronomía. Tesis en opción al grado académico de Master en ciencias de la educación, Instituto Superior Pedagógico Blas Roca Calderío. Granma. 